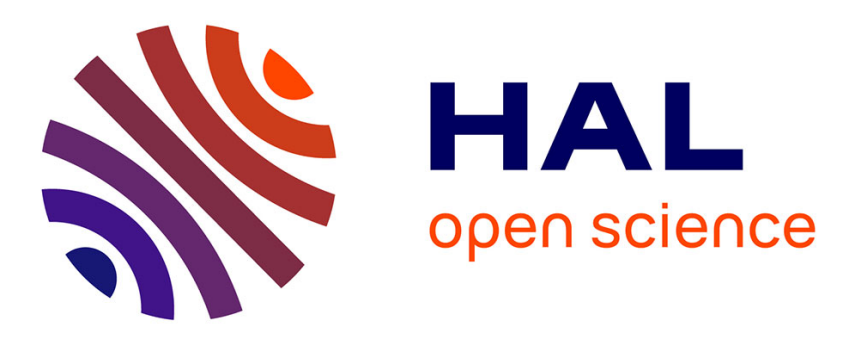

\title{
An Enhanced Deterministic Beacon Advertising Algorithm for Building TSCH Networks
}

\author{
Ines Khoufi, Pascale Minet
}

\section{To cite this version:}

Ines Khouf, Pascale Minet. An Enhanced Deterministic Beacon Advertising Algorithm for Building TSCH Networks. Annals of Telecommunications - annales des télécommunications, 2018. hal01870277

\section{HAL Id: hal-01870277 \\ https://hal.science/hal-01870277}

Submitted on 7 Sep 2018

HAL is a multi-disciplinary open access archive for the deposit and dissemination of scientific research documents, whether they are published or not. The documents may come from teaching and research institutions in France or abroad, or from public or private research centers.
L'archive ouverte pluridisciplinaire HAL, est destinée au dépôt et à la diffusion de documents scientifiques de niveau recherche, publiés ou non, émanant des établissements d'enseignement et de recherche français ou étrangers, des laboratoires publics ou privés. 


\title{
An Enhanced Deterministic Beacon Advertising Algorithm for Building TSCH Networks
}

\author{
Ines Khoufi • Pascale Minet
}

Received: date / Accepted: date

\begin{abstract}
In this paper, we focus first on the time needed by a node to join a Time Slotted Channel Hopping (TSCH) network, this time is called joining time. Second, we are also interested in the network building time. Since the data generated by a sensor node remain unavailable as long as this node has not yet joined the wireless sensor network, these times are of prime importance for applications having strong latency requirements on data gathering. The joining time depends on the beacon advertising policy that has been left unspecified by the standard. The contribution of this paper is triple. First, we propose an Enhanced Deterministic Beacon Advertising algorithm, called EDBA, that ensures a collision-free advertising of beacons and minimizes the average joining time. Second, we model the behavior of a joining node by a Markov chain, validated by NS3 simulations, and compute the average joining time. Third, we compare the performance of EDBA with this of MBS, considered as the best beacon advertising algorithm in the literature.
\end{abstract}

Keywords TSCH network $\cdot$ beacon advertising $\cdot$ node joining time $\cdot$ network building

\section{Introduction}

Wireless Sensor Networks (WSNs) [1] pave the way to a wide range of applications belonging to as various domains as environment monitoring, factory automation, process control, precision agriculture, e-health [2], smart city, vehicular communication, etc. The most frequent technologies used by the currently deployed WSNs are based on the IEEE 802.15.4 standard [3].

Ines Khoufi, Pascale Minet

Inria Paris

ines.khoufi@gmail.com

pascale.minet@inria.fr 


\subsection{Context and motivation}

The Time Slotted Channel Hopping (TSCH) mode has been designed as a part of the IEEE $802.15 .4 \mathrm{e}$ [4] amendment to meet the requirements of latency, throughput and robustness of applications belonging to industrial automation, process control and equipment monitoring. TSCH is the most used mode among the five modes introduced in this amendment (see [5] for a survey of this amendment). In the following of this paper, an IEEE 802.15.4e TSCH network is denoted a TSCH network, for brevity reason.

TSCH provides time-synchronized communications (time slots) and uses different channels in parallel with frequency hopping. Figure 1 illustrates a schedule of transmissions on 16 frequencies using a periodic slotframe of 28 time slots. The transmissions belonging to this schedule are represented by colored rectangles. Each transmission is defined by its transmitter, its receiver(s), its channel offset depicted in the vertical axis and a slot offset in the horizontal axis. The pair (slotoffset, channeloffset) is called cell in this paper. At each slot change, the mapping of channel offsets to real frequencies changes. The ASN of a slot is its Absolute Sequence Number, starting from 0. The slot offset can be deduced from the ASN as follows: slotoffset $=A S N \operatorname{modulo} S_{n}$, where $S_{n}$ denotes the number of slots in the slotframe.

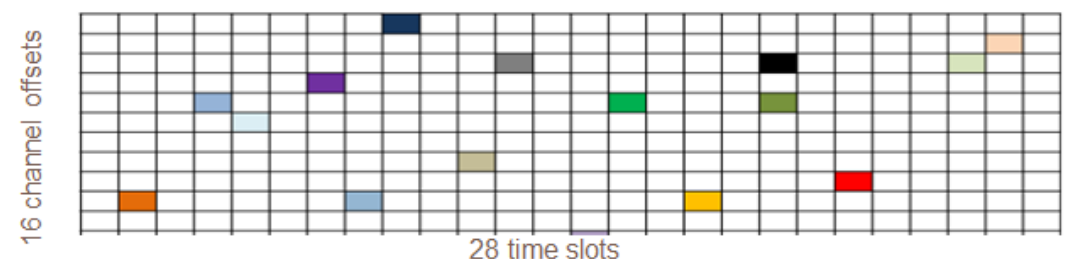

Fig. 1: An example of a TSCH schedule.

Since a multichannel TDMA medium access is used for data gathering, there is no collision on data. Hence, a higher throughput is obtained. In addition, channel hopping significantly reduces the effects of multipath and interferences, improving network robustness. TSCH supports star, tree and meshed topologies.

Unlike most of the studies on IEEE 802.15.4e networks that focus on communications in an operational network, we focus on two times:

- the joining time, defined as the time needed by a node $J$ to join an existing TSCH network. We evaluate this time as the time needed by $J$ to detect a valid beacon.

- the network building time, defined as the time needed to build the network consisting of a given number of nodes that are powered on successively. The network building time is equal to the time at which the last node joins the 
network minus the time at which the CPAN (i.e. the network coordinator) has been powered on.

Both times matter for industrial applications that have strong latency requirements on data gathering. As long as a sensor node has not joined the network, its data remain unavailable and cannot be exploited. A node has to join the network at least once: either when the network is built, or later if the node arrives late or it replaces a failed one. In all cases, the joining node is unable to join the network as long as it has not detected a valid beacon.

Network building is performed by nodes joining the network, successively and/or in parallel. All the solutions proposed to join a TSCH network are based on the following basic principle:

- The joining node randomly selects a frequency among those used by the TSCH network it wants to join. It listens on this frequency until detecting a valid beacon. A beacon is a TSCH frame that contains useful information about synchronization, channel hopping and time slot used in the network considered.

- Nodes having joined the network periodically advertise beacons. Initially, only the CPAN is allowed to send beacons. The presence of the CPAN is mandatory to initiate the creation of the network.

With TSCH, the joining node $J$ has to face a triple problem:

1. First, the beacon advertising policy is voluntarily left unspecified by the standard.

2. Second, the main difficulty with a TSCH network comes from the frequency hopping and the simultaneous use of several channels. How to ensure that any node $J$ that wants to join the network is listening on the frequency used by a network node advertising its beacon and located in the neighborhood of $J$ ?

3. Third, as beacons are broadcast, they may collide. In such a case, the collision remains undetected and the important information they carry is lost. How to avoid collisions between nodes advertising beacons? In [6], the authors propose a distributed algorithm where each node selects the slot to transmit its beacon with a probability depending on the feedback provided by its neighbors. This algorithm is proved to converge almost surely in a finite time. In [7], the authors jointly address beacon synchronization and duty cycling in IEEE 802.15.4 cluster tree networks, and show how to maximize energy saving.

We propose the Enhanced Deterministic Beacon Advertising Algorithm, called EDBA, to solve this triple problem.

This paper is organized as follows. After a brief state of the art, we present the main principles of EDBA and illustrate its behavior with some examples in Section 2. In Section 3, we build a model for a node joining a TSCH network using the EDBA algorithm. We derive the average joining time as a function of the number of nodes present in the network and the number of advertising slots, taking into account the unreliability of wireless links. The performance of 
EDBA is compared in Section 4 with this of MBS [8], the algorithm considered as the best one in the literature. Finally, we conclude in Section 5.

\subsection{Related work}

Since TSCH [9] provides low latency due to its multi-channel and time-slotted medium access, many papers use a TSCH network for data gathering. For example, papers published in [10-12] conclude that TSCH networks can be used to achieve low latency event detection or data gathering with a careful setting of TSCH scheduling parameters. Some authors propose a centralized scheduling for scheduling data transmissions such as in [13], whereas others favor a distributed one such as in [14], [15] and [16]. However, in these studies, the TSCH network is assumed to be established. In this paper, we show how to build the TSCH network in order to reduce the network joining time.

To solve the problem raised by multichannel during network building, some authors like [17] want to reduce the time spent and the energy consumed in this phase. They propose to dedicate a channel to beacons. Hence, a joining node has only this channel to scan, whereas network nodes have to periodically advertise their beacons on this channel. This is not easy to apply in a TSCH network, where the schedule is established for channel offsets that are virtual and not for physical frequencies that are real.

That is why in the state of the art, it is usually assumed that several frequencies are used to advertise beacons. In the Random-based Advertisement (RA) algorithm [18], each network node advertises its beacon in advertising slots with a probability computed from the number of nodes advertising a beacon. The authors show that the joining time mainly depends on the number of channel offsets used for beacon advertisement. A reduced joining time is obtained when only a subset of frequencies is used for beacon advertisement. This subset should be known from the joining nodes.

In [19], the authors present two algorithms: Random Vertical (RV) and Random Horizontal (RH), illustrated in Figures 2a and 2b. In both algorithms, the CPAN sends its beacon in slot offset 0 and channel offset 0 . In RV, the other nodes transmit their beacon in the same slot offset but with a random channel offset. In RH, the other nodes transmit their beacon with channel offset 0 but in an random slot offset. The authors show that these algorithms have similar performance.

In [8], the authors model the behavior of a joining node as a Markov chain. They derive the joining time from this model, assuming that the number of channels available $N_{c}$ and the number of slots in the slotframe $N_{s}$ are coprime (i.e. their greatest common divisor is 1), and for each slot offset in the slotframe, the mapping between the channel offset and the channel frequency is bijective. The optimal beacon schedule is formalized as an optimization problem where the joining time is minimized under the constraint that the sum of the distances between the advertising slots is equal to $N_{c} N_{s}$. The authors finally propose the Model-based Beacon Scheduling algorithm (MBS), 


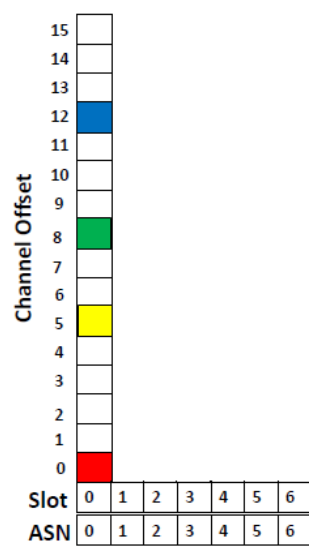

(a) Random Vertical.

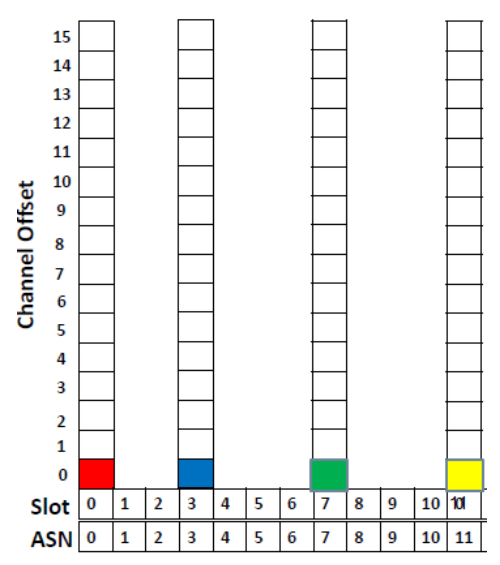

(b) Random Horizontal.

Fig. 2: Random Vertical and Random Horizontal algorithms.

where the CPAN broadcasts the optimal cells (channel offset, slot offset) for beacon advertising. Each network node randomly selects one cell among the optimal cells to transmit its beacon. A comparative performance evaluation is made between MBS, RA, RV and RH. MBS is the best one, even if it is subject to collisions when several nodes transmit their beacon in the same cell.

\section{The Enhanced Deterministic Beacon Advertising algorithm}

Before presenting the Enhanced Deterministic Beacon Advertising algorithm, called EDBA, we define a generic framework to allow nodes to join a TSCH network.

\subsection{General framework}

Before giving the assumptions used in this study, we recall some mathematical results.

Two positive integers are said co-prime if and only if they have no common divisor higher than 1 . Hence their least common multiple is equal to their product.

We adopt the following assumptions:

- A0: For each slot offset in the slotframe, the channel hopping sequence is a bijection from the set of channel offsets to the set of channel frequencies used by the TSCH network considered. In addition, for simplicity reasons, in all the examples taken, this bijection is the identity for slot offset 0 . 
- A1: The slotframe has $N_{s}$ slots.

- A2: The TSCH network uses $N_{c}$ channels.

- A3: $N_{s}$ and $N_{c}$ are coprime and $S$ denotes their product.

- A4: The slotframe contains $N_{b}$ beacon cells.

- A5: The spacing between two successive beacon cells $b_{i}$ and $b_{i+1}$ in $S$ successive slots is equal to:

$A S N\left(b_{i+1}\right)-A S N\left(b_{i}\right)$ if $A S N\left(b_{i+1}\right)>A S N\left(b_{i}\right)$, $A S N\left(b_{i+1}\right)+N_{s} \times N_{c}-A S N\left(b_{i}\right)$ otherwise.

Property 1 The TSCH schedule repeats in time slots and frequencies each $\operatorname{lcm}\left(N_{c}, N_{s}\right)$ slots, where $l c m\left(N_{c}, N_{s}\right)$ denotes the least common multiple of $N_{c}$ and $N_{s}$.

Let us first show why Assumption A3 is required. Let us assume that $N_{c}$ and $N_{s}$ have a common divisor $>1$. In this condition, any given cell $(s, c)$ with $s \in\left[0, N_{s}-1\right]$ and $c \in\left[0, N_{c}-1\right]$ is not mapped on all the $N_{c}$ frequencies, even when considering $S$ successive slots. Since this cell appears $\frac{\operatorname{lcm}\left(N_{c}, N_{s}\right)}{N_{s}}<N_{c}$ times in the $S$ slots considered, it cannot be mapped on the $N_{C}$ frequencies. Hence, the justification of Assumption A3.

Example 1: With $N_{s}=15$ and $N_{c}=6$, only two frequencies are visited.

With the Assumptions A0 to A4, we can restrict our study to $S$ successive slots and prove the following properties:

Property 2 In $S$ successive slots, each cell $(s, c)$, with $s \in\left[0, N_{s}-1\right]$ and $c \in\left[0, N_{c}-1\right]$, is mapped exactly once on each of the $N_{c}$ frequencies.

Proof : We first observe that each cell $(s, c)$ visits each frequency at least once in slots of $A S N=0$ to $S-1$. By contradiction, we assume that a given frequency is visited more than once by a given cell $(s, c)$, this would mean that there is an $A S N \in\left[N_{s}, S-1\right]$ that is mapped on the same frequency as $(s, c)$. This is impossible because of A0 and A3. Hence, the property.

Property 3 In $S$ successive slots, there are $N_{b}$ beacon cells mapped exactly once on each of the $N_{c}$ frequencies.

Proof : In $S$ successive slots, there are $N_{c}$ slotframes. Since there are $N_{b}$ beacon cells per slotframe. We get a total of $N_{b} \times N_{c}$ beacon cells for $S$ successive slots. According to Property 2, each beacon cell is mapped on each frequency used by the network. Hence, the property.

\subsection{Principles of EDBA}

EDBA ensures a fair distribution of the $N_{b}$ beacon slots in the slotframe, as illustrated by the different examples shown in Figure 3. The spacing between two successive beacon slots may be Large of size $L=\left\lceil\frac{N_{s}}{N_{b}}\right\rceil$ or Reduced of size 


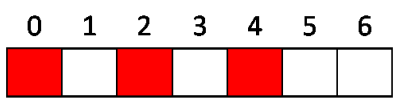

(a) $N_{s}=7$ and $N_{b}=3$, pattern $=2 \mathrm{R}-\mathrm{L}$.

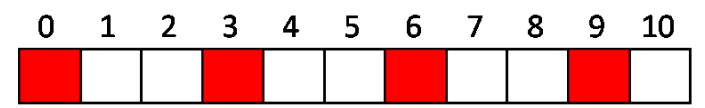

(b) $N_{s}=11$ and $N_{b}=4$, pattern $=3 \mathrm{~L}-\mathrm{R}$.

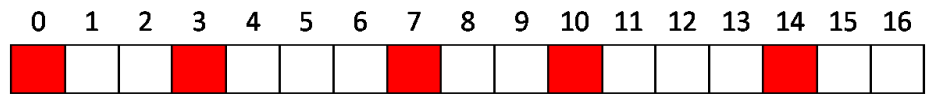

(c) $N_{s}=17$ and $N_{b}=5$, pattern $=2(\mathrm{~L}-\mathrm{R})$.

Fig. 3: Examples of beacon slot assignment.

$R=\left\lfloor\frac{N_{s}}{N_{b}}\right\rfloor$.

In Figure $3 \mathrm{a}$ where $N_{s}=7$ and $N_{b}=3$, Algorithm 1 gives the pattern Reduced-Reduced-Large $=2 R-L$. Then slots 0,2 and 3 are dedicated to beacons advertising. In Figure $3 \mathrm{~b}, N_{s}$ is equal to 11 and $N_{b}$ equal to 4 . Then, the pattern obtained is $3 L-R$ and slots $0,3,6,9$ are beacon slots. The last example is shown in Figure $3 \mathrm{c}$, where $N_{s}=17$ and $N_{b}=5$. Algorithm 1 provides the pattern $2(R-L)$ to determine the beacon slots which are in this case : 0 , $3,7,10,14$.

More precisely, EDBA is based on the following principles:

- Any beacon is transmitted in one of the $N_{b}$ advertising cells of the slotframe.

- The CPAN initiates the beacon transmission in the first advertising cell with channel offset 0 and slot offset 0 .

- The $N_{b}$ advertising cells are regularly spaced in the slotframe according to Algorithm 1. The intuitive idea of this algorithm is first to compute the number of large spacings of size $L=\left\lceil\frac{N_{s}}{N_{b}}\right\rceil$ and the number of reduced spacings of size $R=\left\lfloor\frac{N_{s}}{N_{b}}\right\rfloor$ between two successive beacons in the slotframe, and second to build a pattern from these two types of patterns that is repeated in the slotframe. This is illustrated by the examples shown in Figure 3 .

- There are $u=N_{s}$ modulo $N_{b}$ Large spacings of size $L=\left\lceil\frac{N_{s}}{N_{b}}\right\rceil$;

- There are $N_{b}-u$ Reduced spacings of size $R=\left\lfloor\frac{N_{s}}{N_{b}}\right\rfloor$;

- The beacon slots are assigned according to Algorithm 1. The intuitive idea of this algorithm is first to compute the number of large spacings 
of size $L$ and the number of reduced spacings of size $R$ between two successive beacons

- The CPAN applies an horizontal first policy. It assigns first the cell with the smallest slot offset higher than the slot offset where the correct beacon has been received, modulo the slotframe size. If several exist, it selects the cell with the smallest channel offset.

- Any advertising node, that is not the CPAN, transmits its beacon in a beacon cell computed by the CPAN during the association of the node.

- Multi-hop association ensures the uniqueness of transmitters in this beacon cell.

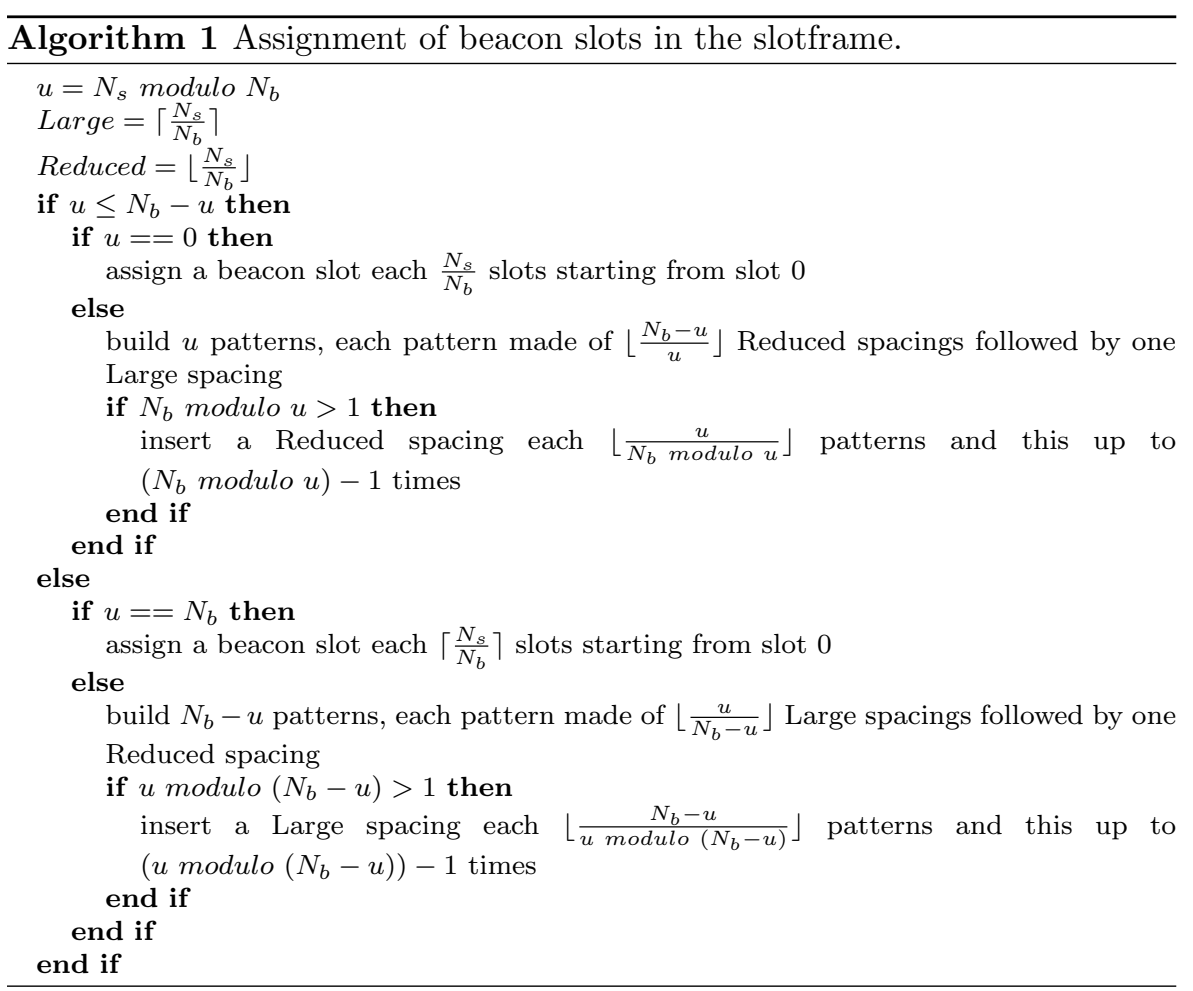

\subsection{Properties of EDBA}

We first establish the condition under which there is no beacon collision with EDBA and then prove some properties.

Condition $1 N_{b} \geq \frac{N_{n}-1}{N_{c}}+1$, where $N_{n}$ denotes the maximum number of neighbors of a node. 
Property 4 With the Condition 1, EDBA ensures the absence of collisions between nodes advertising their beacon.

Proof : In order to enable each node to listen to the beacon sent by the CPAN, no node other than the CPAN transmits its beacon in slot offset 0 . Hence, the total number of beacon cells available is equal to $1+\left(N_{b}-1\right) N_{c}$. As a consequence, the maximum number of neighbors of a node, denoted $N_{n}$ is limited to this number $N_{n} \leq 1+\left(N_{b}-1\right) N_{c}$, leading to $N_{b} \geq \frac{N_{n}-1}{N_{c}}+1$.

Property 5 With the Condition 1, EDBA is deterministic.

Proof : deduced from Property 4.

Property 6 With the Condition 1, the maximum joining time can be computed as a function of $N_{s}, N_{c}$ and $N_{b}$ and the number of neighboring nodes having already joined the network.

Proof : see the computation of the joining time in the next section.

\section{Markov Chain for a joining node with EDBA}

\subsection{Model presentation}

As previously said, a node $J$ wanting to join a TSCH network randomly selects a frequency $f$ among the frequencies used by the TSCH network and listens to this frequency to hear a valid beacon. Since the schedule of a TSCH network repeats in slot offsets and frequencies every $S=N_{c} \times N_{s}$ slots, we consider $S$ successive slots denoted $s_{i}$, with $0 \leq i \leq S-1$. The joining node is powered on at a random time. As a consequence, it has a probability of $1 / S$ to be powered on in any given slot among the $S$ slots considered. The state of the joining node can be modeled by the slot offset in this period, that is $A S N \operatorname{modulo} S$. We denote such a state $s_{i}$, with $0 \leq i \leq S-1$. The behavior of a joining node can be modeled by a discrete time Markov chain shown in Figure 4, where the time granularity is the slot duration.

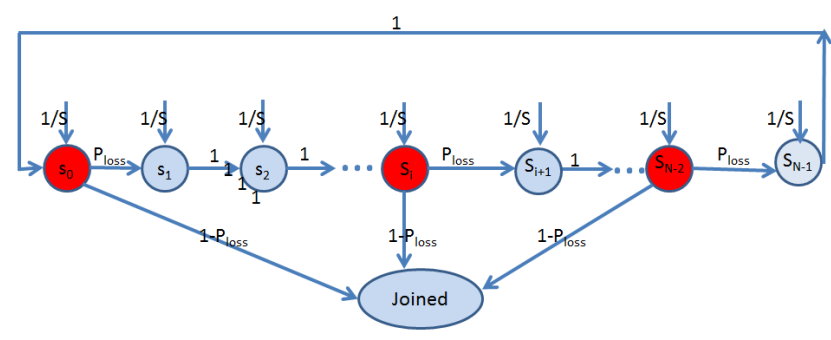

Fig. 4: The Markov model of a joining node with EDBA.

We distinguish whether the slot $s_{i}$ is a beacon slot granted to a neighboring node of the joining node $J$ with a channel offset mapped on frequency $f$. In 
such a case, the slot, also called state, $s_{i}$ is denoted $b_{j}$ and belongs to $\mathcal{B}$, the set of beacon slots assigned to a neighbor of $J$ and mapped on frequency $f$.

The joining node $J$ transits from $s_{i} \notin \mathcal{B}$ to $s_{i+1}$ with a probability 1 corresponding to the transition to the next slot. It transits from $s_{i} \in \mathcal{B}$ to:

- the absorbing state Joined with a probability $1-P_{l o s s}$, where $1-P_{\text {loss }}$ is the probability of receiving a valid beacon sent on the listen frequency $f$.

- the next slot $s_{i+1}$ with a probability $P_{\text {loss }}$.

Unlike the Markov model designed in [8] for MBS, where the number of beacon slots mapped on frequency $f$ in $S$ successive slots is always equal to $N_{b}$, this model takes into account a number of beacon slots that is equal to $N_{n}$ the number of nodes having already joined the network that are neighbors of $J$. Hence, in the Markov model, there are at least $N_{n}$ beacon slots mapped on frequency $f$. As a consequence, the index $j$ in the $b_{j}$ state, ranges from 0 to $N_{n}-1$.

\subsection{Computation of the joining time}

With this model, we are able to compute the average joining time of a node. This time depends on $N_{b}, N_{s}, N_{c}$ and $N_{n}$. Let $\eta_{i}$ denote the number of transitions from any state $s_{i}$ to the Joined state. Let nextb $\left(s_{i}\right)$ denote the next beacon state in $\mathcal{B}$ following $s_{i} \notin \mathcal{B}$. Let $d\left(s_{i}, s_{j}\right)$ denotes the distance between slots $s_{i}$ and $s_{j}$. This distance is expressed by a number of slots. It is equal to: $s_{j}-s_{i}$ if $s_{j} \geq s_{i}$

$s_{j}+N_{s}-s_{i}$ otherwise.

We get:

$$
\begin{aligned}
\eta_{i}= & d\left(s_{i}, n \operatorname{extb}\left(s_{i}\right)\right)+\eta_{\text {nextb(si) }} \text { if } s_{i} \notin \mathcal{B} \\
& \left(1-P_{\text {loss }}\right)+P_{\text {loss }} \eta_{\text {nextb(si) }} \text { otherwise }
\end{aligned}
$$

Let $b_{0}$ denote the first beacon slot in the $S$ successive slots considered and $b_{N n-1}$ the last one. Hence, we have $b_{N n}=b_{0}$. We can deduce the value of $\eta_{0}$, $\eta_{j}$ and finally $\eta_{N_{n}-1}$ as follows:

$\eta_{b 0}=1+\frac{\sum_{i=0}^{N n-1} P_{\text {loss }}^{i} d\left(b_{i}, b_{i+1}\right)}{1-P_{\text {loss }}^{N n}}$

and more generally for $0 \leq j \leq N_{n}-1$

$\eta_{b j}=1+\frac{\sum_{i=0}^{N n-1} P_{l o s s}^{i+N n-j} d\left(b_{i}, b_{i+1}\right)}{1-P_{\text {loss }}^{N n}}+\sum_{i=1}^{N n-j} P_{\text {loss }}^{i} d\left(b_{j+i-1}, b_{j+i}\right)$.

Let $T_{\text {Join }}$ denote the joining time. it is equal to the sum of the numbers of transitions to the Joined state from each state $s_{i}$ divided by $S$ the total number of states.

$$
T_{\text {Join }}=\sum_{i=0}^{S-1} \frac{\eta_{i}}{S}
$$


For reliable wireless links with $P_{\text {loss }}=0$, we obtain $\eta_{i}=1$ for any $s_{i} \in \mathcal{B}$, leading to:

$$
T_{\text {Join }}=\frac{1}{S}\left(N_{n}+\sum_{j=0}^{N n-1} \frac{d\left(b_{j}, b_{j+1}\right)\left(d\left(b_{j}, b_{j+1}\right)-1\right)}{2}\right)
$$

For unreliable wireless links, we get:

$$
T_{\text {Join }}=\frac{1}{S}\left(N_{n}+\sum_{j=0}^{N n-1}\left(\frac{d\left(b_{j}, b_{j+1}\right)\left(d\left(b_{j}, b_{j+1}\right)-1\right)}{2}+\sum_{i=0}^{N_{n}-1} P_{l o s s}^{i+N n-j} d\left(b_{i}, b_{i+1}\right)\right)\right)
$$

\subsection{Model validation}

We assume that the joining node is joining a network consisting of 1 to 10 nodes. The CPAN is denoted node 0 . The node $i$ joins the network consisting of nodes 0 to $i-1$. This joining time is expressed as a number of slots, each slot having a duration of $10 \mathrm{~ms}$. We report here the joining time obtained for $N_{c}=5, N_{s}=3$ and $N_{b}=3$ to highlight the differences with the model of MBS given in [8]. For this example, we assume the simple frequency mapping given in Table 1. In this table slof denotes the slot offset whereas chof denotes the channel offset. Results are given in Table 2, where a star denotes a beacon slot. In Table 1 and Table 2, ASN modulo $N_{s} N_{c}$ is denoted Am.

\begin{tabular}{|c||c|c|c|c|c|c|c|c|c|c|c|c|c|c|c|}
\hline slof & 0 & 1 & 2 & 0 & 1 & 2 & 0 & 1 & 2 & 0 & 1 & 2 & 0 & 1 & 2 \\
\hline$A m$ & 0 & 1 & 2 & 3 & 4 & 5 & 6 & 7 & 8 & 9 & 10 & 11 & 12 & 13 & 14 \\
\hline \hline chof0 & 0 & 1 & 2 & 3 & 4 & 0 & 1 & 2 & 3 & 4 & 0 & 1 & 2 & 3 & 4 \\
\hline chof1 & 1 & 2 & 3 & 4 & 0 & 1 & 2 & 3 & 4 & 0 & 1 & 2 & 3 & 4 & 0 \\
\hline chof2 & 2 & 3 & 4 & 0 & 1 & 2 & 3 & 4 & 0 & 1 & 2 & 3 & 4 & 0 & 1 \\
\hline chof3 & 3 & 4 & 0 & 1 & 2 & 3 & 4 & 0 & 1 & 2 & 3 & 4 & 0 & 1 & 2 \\
\hline chof4 & 4 & 0 & 1 & 2 & 3 & 4 & 0 & 1 & 2 & 3 & 4 & 0 & 1 & 2 & 3 \\
\hline
\end{tabular}

Table 1: Frequency mapping.

When the CPAN is the only node in the network, it sends its beacon in slot offset 0 and channel offset 0 by design of EDBA. According to Table 1, this cell is mapped on frequency 0 for $A S N=0$ modulo $N_{s} N_{c}$. The number of transitions to the Joined state is equal to 1 for this ASN and is computed according to Equation 1 for the other ASNs. Hence, the third column of Table 2. When node 1 is inserted, it sends its beacon in slot offset 1 and channel offset 0 , according to EDBA. According to Table 1, this cell is mapped on frequency 0 for $A S N=10 \operatorname{modulo} N_{s} N_{c}$. The number of transitions to the Joined state is equal to 1 for this $\mathrm{ASN}$ and is computed according to Equation 1 for the 


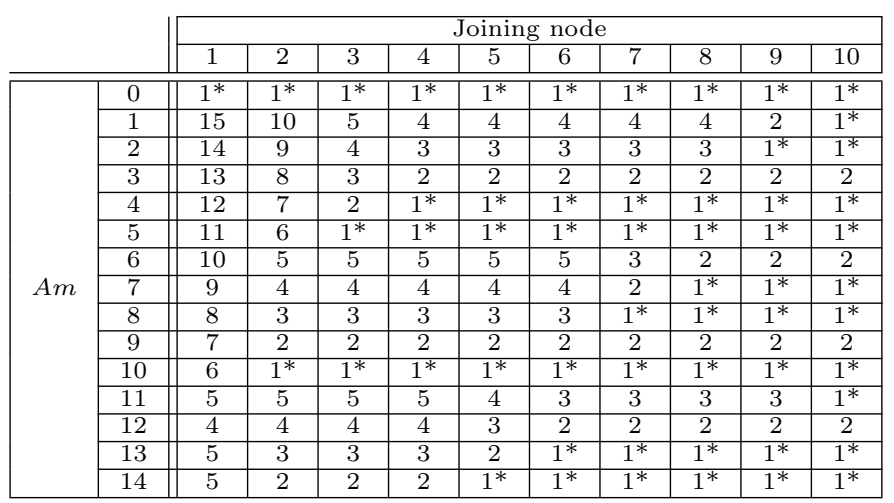

Table 2: Number of transitions to the Joined state.

other ASNs. We get the fourth column of Table 2, and so on.

This model has been validated by means of simulations with NS3 [20]. We first implemented the NS3 modules needed to build a 802.15.4 network and then the NS3 modules for a TSCH network. Each simulation result reported in this paper is the average of 200 simulations. We compute the average, the standard deviation and the $95 \%$ confidence interval of these simulations runs and represent the average and the error bars in all figures showing the simulations results. We compare the average joining time obtained by computation based on the model and by simulation, when $N_{n}$ neighbors of the joining node send beacons, with $1 \leq N_{n} \leq 10$. For the model, the average joining time is obtained as the average of each column of Table 2. Figure 5 shows the average joining time obtained with the model and the NS3 simulations for a number of neighbors $N_{n}$ ranging from 1 to 10 . Simulation results corroborate the model results. Hence, the Markov model is validated.

\subsection{Main differences with MBS}

We now compare the joining time obtained by EDBA and MBS, when the number of nodes advertising their beacon ranges from 1 to 10 , assuming a one-hop network and taking the values $N_{c}=5, N_{s}=3$ and $N_{b}=3$ adopted in [8]. The joining times are illustrated in Figure 6. When the number of advertising nodes is less than or equal to 2, MBS and EDBA obtain the same joining time. With EDBA, the joining time continuously decreases when the number of advertising nodes increases up to 10 . This is not the case with MBS. The reason is due to the collisions, since the advertising nodes randomly select one of the two beacon cells, the third one being reserved to the CPAN. Notice that to avoid an unbounded waiting time for the joining node in the MBS simulations, the beacon cell of the CPAN is not shared with other nodes. As a 


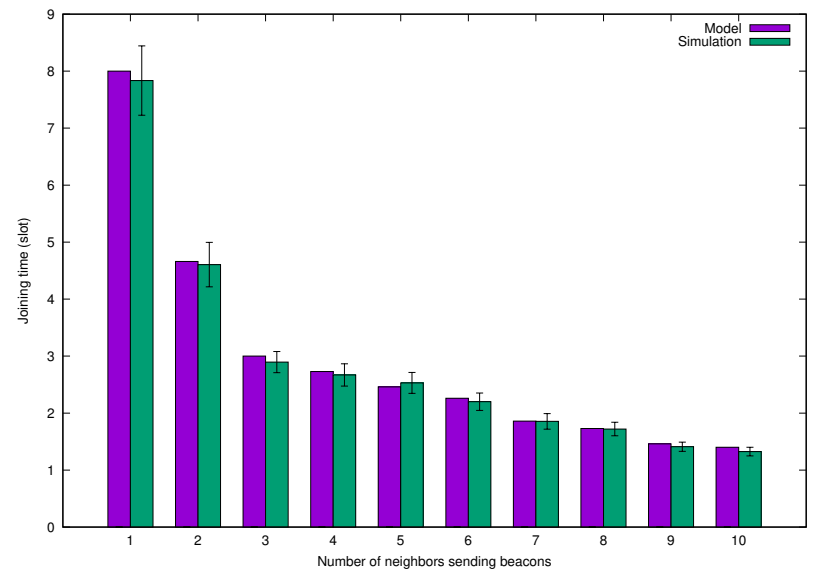

Fig. 5: Validation of the Markov model by NS3 simulations.

consequence, the joining time is upper-bounded by $N_{s} N_{c}=15$ slots for MBS, whereas it is much smaller for EDBA as shown in Figure 6.

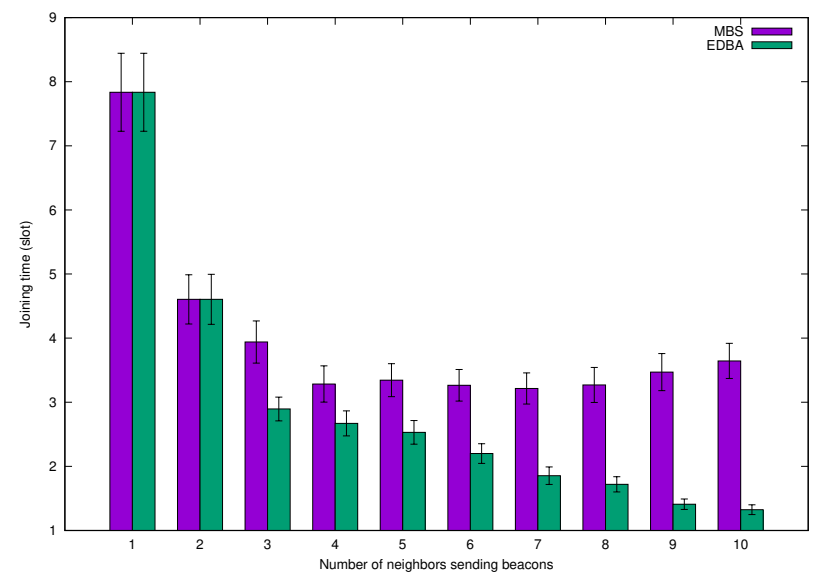

Fig. 6: Joining time obtained by EDBA and MBS.

\section{Comparative performance evaluation}

In this section, our goal is to evaluate the time needed to join the network and then to build the network, as well as the number of beacons transmitted and the number of beacons dropped due to collisions. We compare the performance of EDBA with this of MBS [8], assuming that the nodes join the network one 
after the other. We start by considering reliable wireless links where beacons are transmitted with a loss probability $P_{\text {loss }}=0$. Then, we consider unreliable wireless links where beacons are lost with probability $P_{\text {loss }} \in\{10 \%, 20 \%, 30 \%\}$. The performance evaluation is conducted using the NS3 simulator. The NS3 simulator allows us to take into account the communication protocols used in real TSCH networks. With simulation, we are also able to compare the solutions evaluated in more detailed way. For instance, we can compute the number of lost beacons. The topology considered is a star topology where each node is in radio range of each other. The number of nodes range from 1 to 40 . The number of channels is the default one $N_{c}=16$, the number of slots in the slotframe $N_{s}$ is equal to 101. All the nodes successively join the network in the order of their identifier. Hence, when node $i \in[2,40]$ joins the network, all the nodes with an identifier strictly less than $i$ advertise their beacon.

\subsection{Reliable wireless links}

In this subsection, the wireless links are assumed to be reliable. Figures 7 , 8 and 9 illustrate the joining time obtained by MBS and EDBA when the number of beacon cells in the slotframe $N_{b}$ is equal to 5,10 or 15 respectively. When the number of advertising nodes is low, the joining time is high for both MBS and EDBA. This joining time decreases when both the number of advertising nodes and the number of beacon cells in the slotframe increase. When the number of advertising nodes is strictly less than $N_{b}$, MBS may provide a smaller joining time than EDBA. This is explained by the design of MBS where each advertising node randomly selects a cell among the $N_{b}$ cells in each slotframe to advertise its beacon and these cells are fairly distributed among $S$ successive slots, whereas EDBA uses a number of beacon cells that is equal to the minimum between $N_{b}$ and the number of advertising nodes. However, EDBA offers a much smaller joining time than MBS, as soon as the number of advertising nodes is higher than the number of beacon cells in the slotframe. For instance, when the number of advertising nodes is 40 , the average value of joining time is 20.7 for EDBA and 104.3 for MBS. With MBS, we observe an increased time needed to join the network in this case, due to collisions. As soon as each beacon cell, except this reserved for the CPAN, is selected by more than one node, a node that wants to join the network has to wait for the next beacon from the CPAN. Then, the maximum joining time is equal to $N_{c}$ times the size of the slotframe.

Figure 10 shows for each joining node, the average number of beacons transmitted since the previous node started its beacon detection, for $N_{b}=5$, 10 and 15 . In other words, the number of beacons sent between two successive node insertions. EDBA is a deterministic solution that avoids beacon's collision. However, many beacons may collide with MBS since this solution is based on a random selection of the advertising cells. This explains why with EDBA, this number remains stable: the node that wants to join the network promptly detects a beacon. With MBS, the number of beacons tends to in- 


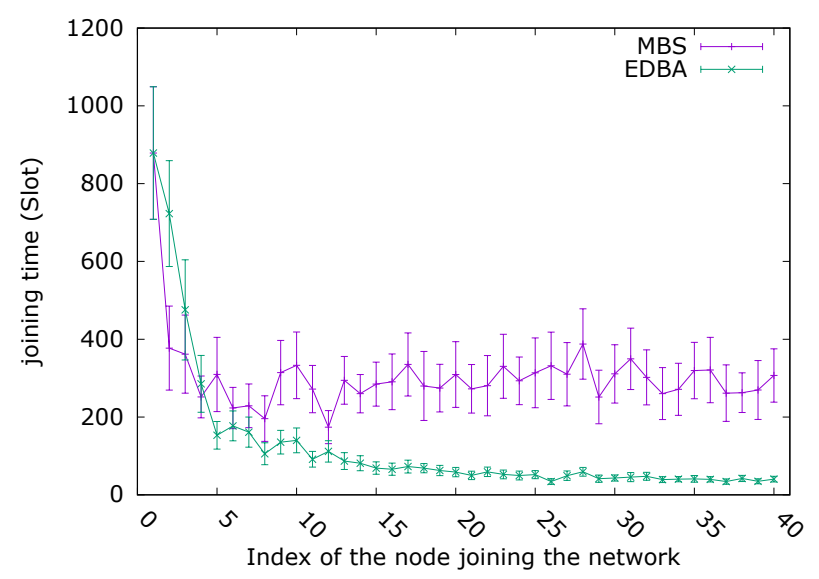

Fig. 7: The joining time obtained by MBS and EDBA for reliable links $\left(N_{b}=5\right)$.

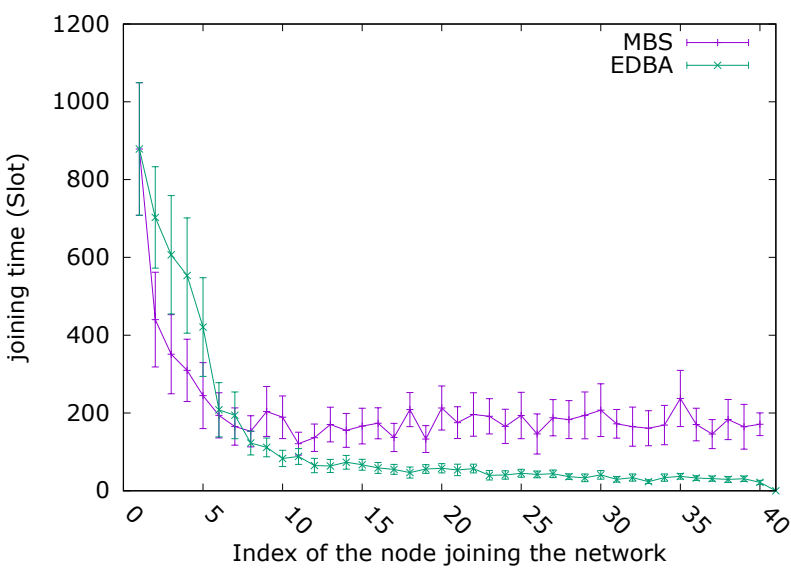

Fig. 8: The joining time obtained by MBS and EDBA for reliable links $\left(N_{b}=10\right)$.

crease with the number of advertising nodes due to the increased number of collisions.

Figure 11 shows the average number of beacons dropped for $N_{b}=5,10$ and 15 for both EDBA and MBS. Since EDBA is deterministic, the number of beacons dropped is 0 . However, with MBS, this number increases when the number of advertising nodes increases. This is due to the random selection of beacon cells that leads to collisions. 


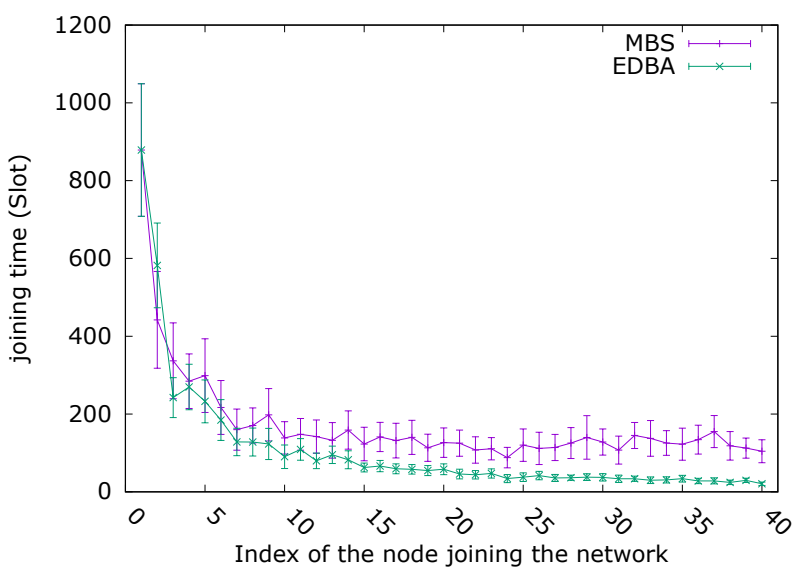

Fig. 9: The joining time obtained by MBS and EDBA for reliable links $\left(N_{b}=15\right)$.

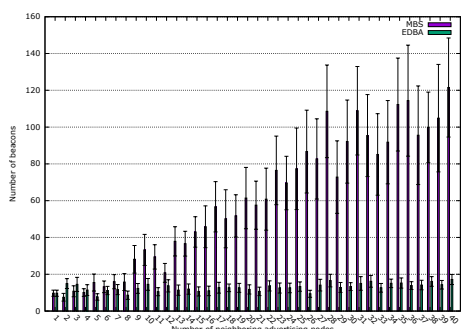

(a) $N_{b}=5$.

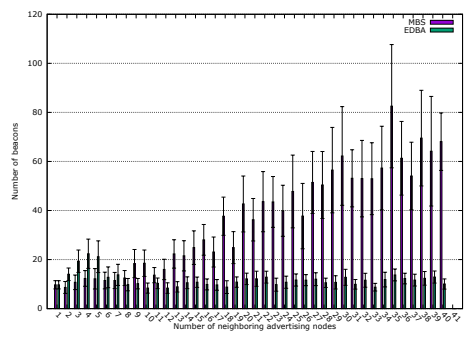

(b) $N_{b}=10$.

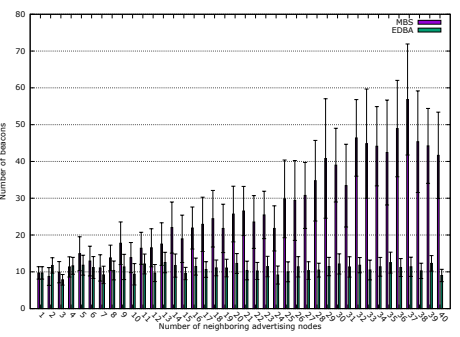

(c) $N_{b}=15$.

Fig. 10: Average number of beacons transmitted

\subsection{Unreliable wireless links}

We now consider unreliable wireless links where beacons are lost with a probability $P_{\text {loss }} \in\{10 \%, 20 \%, 30 \%\}$. In this subsection, we perform 200 simulation runs to obtain accurate results when unreliable wireless links are consid- 


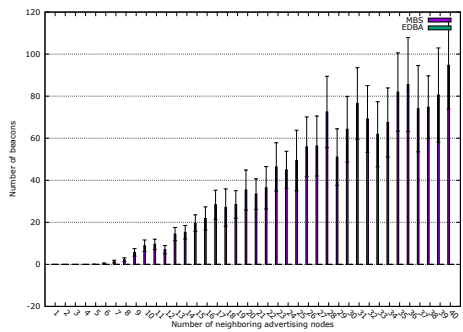

(a) $N_{b}=5$.

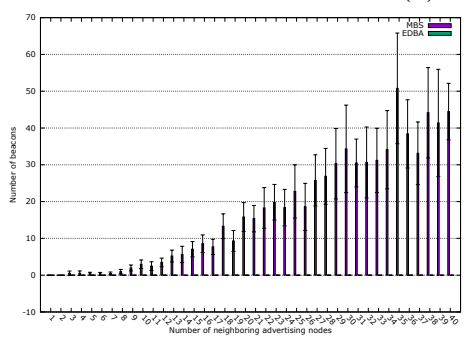

(b) $N_{b}=10$.

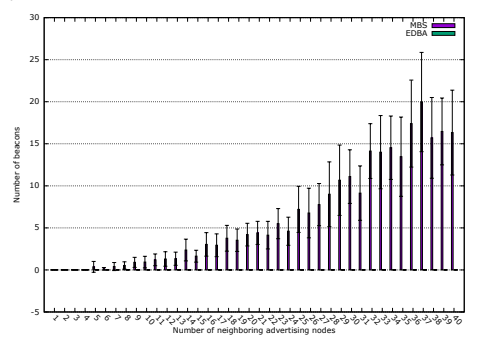

(c) $N_{b}=15$.

Fig. 11: Average number of beacons dropped

ered. We evaluate the node joining time and the network building time when $N_{b}=10$.

In Figure 12, we illustrate the joining time of each node. When unreliable wireless links are considered, the time the node waits until it detects a beacon is very large. This is due to, first, the few number of advertising nodes, second, beacons loss. When unreliable wireless links are considered and the number of advertising nodes increases, the node joining time is still high with MBS, however, with EDBA it becomes very close to the value obtained in case of reliable wireless links.

Table 3 gives the cumulative network joining time for both solutions EDBA and MBS in terms of slots. This cumulative joining time can also be considered as the network building time, when nodes successively join the network. When wireless links are reliable, we can see that with EDBA the network building time is $36 \%$ less than the time provided by MBS.

In case of unreliable wireless links, the time needed to build the network considerably increases for both solutions. However, EDBA still performs better than MBS. This can be explained by the fact that with MBS beacons can be lost not only because of unreliable wireless links but also collisions. Hence the very large delay to build the network. However, with EDBA, we can lose beacons when wireless links are unreliable but there is no beacon collision due to its deterministic principle. 


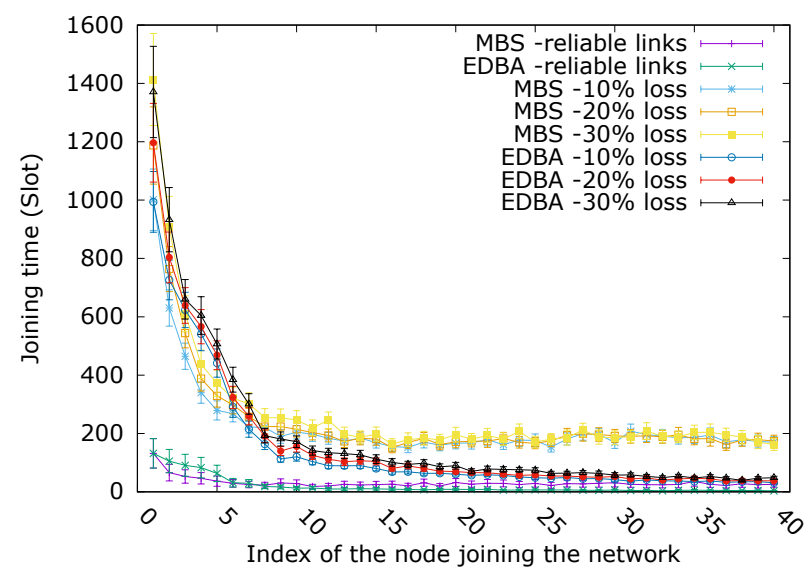

Fig. 12: Average joining time (slot) for $N_{b}=10$.

\begin{tabular}{|c|c|c|c||c|c|c|c|}
\hline \multicolumn{3}{|c||}{ EDBA } & \multicolumn{4}{c|}{ MBS } \\
\hline Reliable & \multicolumn{3}{|c|}{ Unreliable } & Reliable & \multicolumn{3}{c|}{ Unreliable } \\
\hline & $10 \%$ & $20 \%$ & $30 \%$ & & $10 \%$ & $20 \%$ & $30 \%$ \\
\hline \hline 796 & 5916 & 6726 & 7659 & 1257 & 9208 & 9907 & 10908 \\
\hline
\end{tabular}

Table 3: Network building time for reliable and unreliable wireless links (slots) for $N_{b}=10$

Figure 13 shows the network building time for both MBS and EDBA. In this figure, we can see the time needed to insert an additional node in the network as a function of neighboring nodes having already joined the network. EDBA and MBS provide close results when the number of nodes is small. However, when the number of nodes increases, EDBA performs better than MBS.

\section{Conclusion}

The optimization of the time needed by a node to join a TSCH (Time Slotted Channel Hopping) network led us to specify the advertising beacon policy. We proposed the EDBA (Enhanced Deterministic Beacon Advertisinq) algorithm. This algorithm ensures that each node advertises its beacon without collision. The beacon cells are fairly distributed in the slotframe. The behavior of a joining node has been modeled by a Markov chain from which the average joining time is computed, taking into account the reliability of wireless links. An intensive performance evaluation based on NS3 simulations allows us to validate this model and conclude on the very good performance of EDBA, even when compared with MBS, considered as the best advertising algorithm in the literature. 


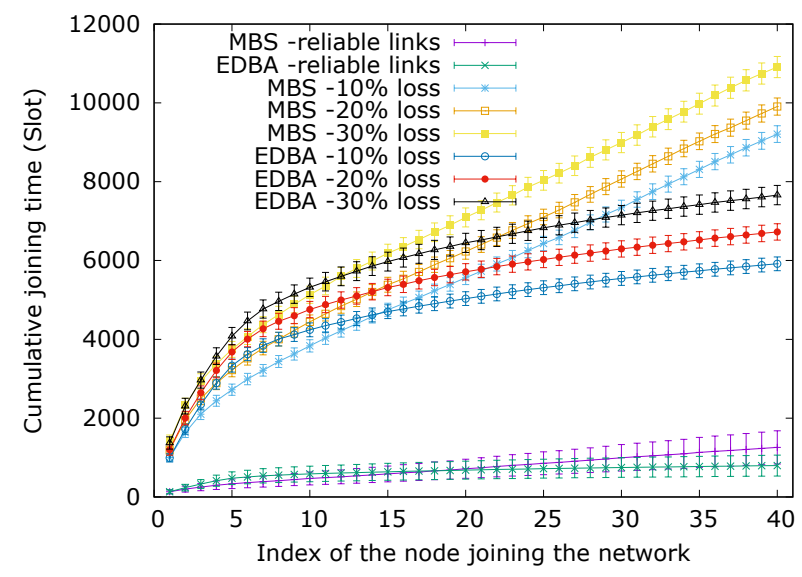

Fig. 13: Average building time (slot) for $N_{b}=10$.

\section{Acknowledgment}

Study co-funded by CNES and Inria in the framework of the CNES Launchers Research and Technology program.

\section{References}

1. A. Derhab, M. Guerroumi, and M. Younis, "Wireless and mobile sensing technologies for the future internet," Annals of Telecommunications, vol. 72, no. 3, pp. 117-118, Apr 2017. [Online]. Available: https://doi.org/10.1007/s12243-017-0570-y

2. A. Moravejosharieh and J. Lloret, "Performance evaluation of co-located ieee 802.15.4based wireless body sensor networks," Annals of Telecommunications, vol. 71, no. 9, pp. 425-440, Oct 2016. [Online]. Available: https://doi.org/10.1007/s12243-016-0497-8

3. IEEE SA, "IEEE Standard for Local and metropolitan area networks-Part 15.4 Low-Rate Wireless Personal Area Networks (LR-WPANs)," IEEE, IEEE Std 802.15.4-2011 (Revision of IEEE Std 802.15.4-2006), Sept 2011. [Online]. Available: http://dx.doi.org/10.1109/ieeestd.2006.232110

4. - " "IEEE Standard for Local and metropolitan area networks-Part 15.4: Low-Rate Wireless Personal Area Networks (LR-WPANs) - Amendment 1: MAC sublayer," IEEE, IEEE Std 802.15.4e-2012 (Amendment to IEEE Std 802.15.4-2011), February 2012 [Online]. Available: http://standards.ieee.org/getieee802/download/802.15.4e-2012.pdf

5. D. De Guglielmo, S. Brienza, and G. Anastasi, "IEEE 802.15.4e: A survey," Computer Communication 2016, vol. 88, pp. 1-24, 2016.

6. C. Cano and D. Malone, "A Learning Approach to Decentralised Beacon Scheduling," in Ad-Hoc Networks Journal, vol. 49. Elsevier, 2016.

7. N. Choudhury, R. Matam, M. Mukherjee and L. Shu, "Beacon Synchronization and Duty-Cycling in IEEE 802.15.4 Cluster-Tree Networks: A Review," in IEEE Internet of Things Journal, 2018.

8. D. De Guglielmo and S. Brienza and G. Anastasi, "A Model-based Beacon Scheduling algorithm for IEEE 802.15.4e TSCH networks," in 2016 IEEE 17th international symposium on a World of Wieless, Mobile and Multimedia Networks (WoWMoM), Coimbra, Portugal, 2016. 
9. T. Watteyne, V. Handziski, X. Vilajosana, S. Duquennoy, O. Hahm, E. Baccelli and A. Wolisz, "Industrial wireless IP-based cyber-physical systems," in Proceedings of the IEEE, vol. 104, no. 5, 2016.

10. I. Khoufi, P. Minet and B. Rmili, "Scheduling transmissions with latency constraints in an IEEE 802.15.4e TSCH network," in IEEE 86th Vehicular Technology Conference (VTC 2017-Fall), Toronto, Canada, 2017.

11. S. Rekik, N. Baccour, M. Jmaiel and K. Drira, "A Performance Analysis of Orchestra Scheduling for TSCH network," in Internet Technology Letters. Wiley, 2017.

12. A. Yang, A. Sundararajan, C. Schindler and K. Pister, "Analysis of Low Latency TSCH Networks for Physical Event Detection," in WCNC 2018, IEEE Wireless Communications and Networking Conference, Barcelona, Spain, 2018.

13. R. Soua, P. Minet, and E. Livolant, "MODESA: An optimized multichannel slot assignment for raw data convergecast in wireless sensor networks," in IPCCC 2012 : 31st IEEE International Performance Computing and Communications Conference. Austin, Texas, United States: IEEE, Dec. 2012, pp. 91 - 100. [Online]. Available: https://hal.archives-ouvertes.fr/hal-00863360

14. —_ "Wave: A distributed scheduling algorithm for convergecast in ieee 802.15.4e tsch networks," Trans. Emerg. Telecommun. Technol., vol. 27, no. 4, pp. 557-575, Apr 2016. [Online]. Available: https://doi.org/10.1002/ett.2991

15. N. Accettura, E. Vogli, M. Palattella, L. Grieco, G. Boggia, and M. Dohler, "Decentralized traffic aware scheduling in 6tisch networks: Design and experimental evaluation," vol. 2, 122015 .

16. M. Domingo-Prieto, T. Chang, X. Vilajosana, and T. Watteyne, "Distributed pid-based scheduling for 6tisch networks," IEEE Communications Letters., vol. 20, no. 5, pp. 1006-1009, May 2016.

17. P. Minet, G. Chalhoub, E. Livolant, M. Misson, R. Soua, R. Diab, B. Rmili, and J. Perelgritz, Wireless Sensor Systems for Extreme Environments: Space, Underwater, Underground and Industrial. West Sussex, UK: John-Wiley, 2016, ch. Multichannel Wireless Sensor Networks for Structural Health Monitoring of Aircraft and Launchers.

18. D. De Guglielmo and A. Seghetti and G. Anastasi and M. Conti, "A performance analysis of the network formation process in IEEE $802.15 .4 \mathrm{e}$ TSCH wireless sensor/actuator networks," in 2014 IEEE Symposium on Computers and Communications (ISCC), Madeira, Portugal, 2013.

19. E. Vogli and G. Ribezzo and L. Alfredo Grieco and G. Boggia, "Fast Join and Synchronization Schema in the IEEE 802.15.4e MAC," in 2015 IEEE Wireless Communications and Networking Conference (WCNC) - Workshop - Energy Efficiency in the Internet of Things, New Orleans, LA, 2015.

20. https://www.nsnam.org/, "NS3 a discrete-event network simulator for internet systems. online." 